\section{Nuclear Cardiac Imaging: Principles and Applications}

\author{
A.E. Iskandrian and E.V. Garcia, Eds.
}

New York, NY: Oxford University Press, 2016, 746 pages, $\$ 275$

Cardiovascular imaging is a rapidly changing field. Although the nuclear imaging modality is relatively well established-more than recent CT and MR technology — both its technology and its clinical evidence have advanced considerably in recent years. Thus, continuing education to keep up with the continuing changes is inevitable for nuclear cardiologists, who are the primary target audience of Nuclear Cardiac Imaging: Principles and Applications, although it will also appeal to medical students; residents in cardiology, radiology, or nuclear medicine; and physicians in other specialties.

As the editors mention in the preface, the basic purpose of the book is to provide the most comprehensive and definitive source of detailed and precise information on nuclear cardiac imaging. Another key purpose appears to be delivery of clear clinical information. Its five sections cover historical, technical, and physiologic considerations; diagnosis and risk assessment; the role of nuclear imaging beyond coronary artery disease; advances in cardiac imaging; and challenges for nuclear cardiology.

The first section presents the basic technology and physiology underlying nuclear cardiac imaging, as well as chapters on instrumentation, image acquisition, and image processing and quantification. The reader learns about the mechanisms and methods of SPECT, PET, and gated imaging and how to differentiate image artifacts. Additionally covered are radiation safety, radiopharmaceutical characteristics, and physiology for pharmacologic stress testing.

The second section-the main portion of the book-covers myocardial perfusion imaging, discussing its effectiveness and role in coronary artery disease based on evidence from clinical studies. Because each chapter deals with a specific clinical setting, such as diabetes, chronic kidney disease, screening, and preoperative risk assessment, the extent of information presented will be quite helpful to clinical practitioners.

The third and fourth sections discuss uses other than myocardial perfusion imaging — uses that are important but are not yet widespread in clinical practice or may still be at the research stage. These include imaging of energy metabolism (glucose and fatty acid), sympathetic innervation, myocardial inflammation, and molecular targets. Additionally, the use of artificial intelligence is discussed. Thus, a reader may learn of the future prospects for nuclear cardiac imaging.

The fifth section is the intriguing one, as it deals with current challenges and hot issues that are considered limitations of nuclear cardiac imaging. For example, the topics of increasing costs and radiation exposure are discussed in terms of cost-effectiveness and appropriate-use criteria.

This book is now in its fifth edition, which is the first update since 2008. Compared with previous editions, the most notable highlight is the revised list of authors, almost all of whom are well-known experts and authorities in their fields. It was a pleasant surprise to meet with so many authoritative names in a single book, with the quality of each chapter matching the authors' reputations.

Another notable highlight is the structure and design of the book, whose organization appears to be nearly perfect. Each of the 35 chapters is introduced by a 9- or 10-sentence "key points" section summarizing and outlining the chapter. Some chapters conclude by presenting cases to illustrate clinical applications, and some of these case presentations include a quiz, which may particularly interest beginners in the field.

A final highlight is the many figures of high aesthetic quality. These include case images of nuclear cardiology, as well as colorful schematic illustrations. Additionally, some supplemental online content and video clips are provided. The endeavor to provide high-quality figures should be noted because of their great helpfulness to the reader.

The only limitations to be pointed out are some minor issues such as the quality of the tables, compared with the quality of figures. The use of color in the design of the tables would be an improvement. In addition, although the book targets chiefly clinicians, the explanation of tracer kinetics (particularly for perfusion PET) and processing algorithms appears to be insufficient. A more detailed mathematic discussion would be better.

This book appears to be one of the best ways for a beginner to learn nuclear cardiac imaging. The text is comprehensive and concise yet is easy to read and understand. Every concept and topic is explained well, and understanding is enhanced by the clear key points and the many tables and beautiful color figures. Almost all the important developments and innovations of recent years are covered. I highly recommend this book not only to beginners but to all in the fields of nuclear medicine, cardiology, radiology.

\section{Jin Chul Paeng \\ Seoul National University Hospital 101 Daehak-ro, Jongno-gu Seoul, 03080, Korea E-mail: paengjc@snu.ac.kr}

Published online May 12, 2016.

DOI: 10.2967/jnumed.116.178137 